\title{
Minimally Invasive Colorectal Surgery and Beyond: A Large, Single-Surgeon Comparison Study Between Robotic and Laparoscopic Colorectal Resection to Evaluate and Define the Role and Application of New Technology
}

\author{
Sara Moncrief ${ }^{1}$, Brano Djenic ${ }^{1 *}$, John Davis ${ }^{1}$, Joel VanderVelde ${ }^{1}$, Salima Hasham ${ }^{1}$ and Andrew Kassir ${ }^{2}$ \\ ${ }^{1}$ Department of General Surgery, Maricopa Integrated Health System, United States \\ ${ }^{2}$ Colon and Rectal Surgery of Scottsdale, United States
}

Submission: May 30, 2017; Published: June 12, 2017

*Corresponding author: Brano Djenic, Department of Surgery, Maricopa Medical Center, Phoenix, AZ 85008, USA, Tel: 2108630673;

Email: brano.djenic@mihs.org

\begin{abstract}
Objective: The use of the daVinci ${ }^{\circledR}$ robot for colorectal resection is increasing. The current literature comparing robotic versus laparoscopic colorectal surgery is limited. Our study addresses the safety, benefits and future applications of the new technology.

Methods: We conducted a retrospective review of prospectively collected data on a consecutive series of patients who underwent either laparoscopic or robotic colon resection in one teaching hospital. We included all patients from April, 2009 to October, 2011 with benign and malignant disease. Data including patient demographics, type and length of procedure, leak rates, conversion rates and complication rates were gathered. Number of lymph nodes and margins were assessed for malignant lesions. We compared the length of the procedure early in our experience to the latter cases.
\end{abstract}

Results: A total of 243 patients were evaluated, 121 LR and 122 RR. The average age was slightly higher in the LR (LR 59.7 vs. 64.4 , $\mathrm{p}=0.007)$. The sex distribution was similar $(\mathrm{p}=0.52)$ and there was no difference in the prevalence of previous operations $(\mathrm{p}=0.19)$. There were no differences in complication rates ( $6.7 \%$ for RR and 10.8 for $\mathrm{LR}, \mathrm{p}=0.36$ ). The anastomotic leak rates were nearly equal (LR $1.7 \%$ vs RR $1.6 \%$, $\mathrm{p}=0.53)$. The number of lymph nodes harvested were similar between both RR and LR groups $(16.8+/-6.6$ vs. $18.1+/-10.2$, $p=0.44)$. There were four conversions to open, all in the RR (1.9\%). Resections for malignancies was greater in the LR group ( $\mathrm{p}=0.001)$. The average operating room time for the RR was longer compared to LR $(151.6 \mathrm{~min}$ vs. $114 \mathrm{~min}, \mathrm{p}=0.0001)$. The operating room time for the first 30 cases averaged 189 minutes while the last 30 cases averaged 137 minutes ( $\mathrm{p}=0.0001)$.

Conclusion: Our data confirms safety and efficacy of robotic resection for benign and malignant colon resection. The use of the robot is not only an acceptable alternative to laparoscopy but demonstrates superior technical advantages in rectal and selective sigmoid resections. A randomized, multi-center prospective analysis is encouraged. Long-term follow up is required to evaluate the survival advantage for malignant pathology and potential benefit of improved pelvic nerve preservation.

\section{Background}

The use of the daVinci ${ }^{\circledR}$ robot for colorectal resection is increasing, however, the current literature comparing robotic and laparoscopic colorectal surgery is limited. Minimally invasive surgery for colorectal disease was introduced in 1991; since then, robotic surgery has been increasingly used worldwide and has gained popularity across surgical subspecialties [1]. Transition from laparoscopy to robotic surgery is well underway,

but strong and convincing data are difficult to find. Some studies have already proven that robotic colorectal surgery is associated with certain benefits compared to conventional laparoscopic colorectal surgery [2-4], however, the relative merits of robotic surgery versus laparoscopic surgery remain controversial. The aim of our study is to compare the new, robotic colorectal surgical technique to the standard laparoscopic technique in terms of safety and efficacy for colorectal resection of benign and 
malignant colorectal disease, to identify technical advantages in colon and rectal benign and malignant resections, and to ultimately encourage large, prospective, randomized studies to compare the overall cost-effectiveness, oncological differences and overall survival.

\section{Materials and Methods}

\section{Study Design}

After Institutional Review Board (IRB) approval, retrospective data collection was completed on prospectively collected patient data on 243 patients who underwent robotic resection $(n=121)$ utilizing the da Vinci SI system (Intuitive Surgical, Sunnyvale, $\mathrm{CA})$ or conventional laparoscopic $(\mathrm{n}=122)$ colon at one hospital system for either colorectal malignancies or pre-malignant colon lesions. Data was obtained from electronic hospital records and follow-up clinical notes. Procedures were performed by a single colorectal surgeon between 2007 and 2012.

\section{Study Inclusion}

Reviewed patient data includes: age, gender, ethnicity, past medical history, past surgical history, ASA classification, body mass index and operative diagnosis. Reviewed case data includes primary procedure performed, length of procedure, complications associated with the disease process, amount of intravenous fluids given during the procedure, estimated blood loss, time at the robotic console, length of hospital stay, postprocedure complications ,pathological specimen diagnosis, and the need for subsequent operative procedures. For cases where the indication for the procedure was resection for malignancy, the number of lymph nodes and margins were assessed. Robotic (RR) and laparoscopic (LR) resections were separated based on indication: malignancy versus pre-malignancy. Some procedures, in both LR and RR approaches, required additional procedures which were inherent to the procedure or planned pre-operatively that did not result in exclusion from the study.

\section{Statistical Analysis}

Data underwent statistical analysis performed by a statistician. One-way analysis of variance, chi-squared test, Fisher's exact test, and two sample t-test with and without unequal variance were calculated using computerized software. Statistical significance was considered to be a P value of $<0.05$.

\section{Results}

Of the 243 consecutive patients who had colon resections from 2009-2012, 121 cases were performed robotically (RR) and 123 laparoscopically (LR). Table 1 shows the average age was slightly higher with LR (LR 59.7 vs. RR 64.4, p=0.007). Comorbidities in two groups included hypertension, gastroesophageal reflux, obesity, endocrine disorders, lung disease, cardiac disease and psychiatric disorders, and we not statistically different. Conversion of the intended operation to a different approach, type of operation performed, all complication rates, resection indications (malignancy or pre-malignant lesion), number of lymph nodes dissected, and average operating room time were compared for each group.

Table 1: RR vs. LR Demographics.

\begin{tabular}{|c|c|c|c|}
\hline & RR & LR & P value \\
\hline Number of Cases & 122 & 121 & -- \\
\hline Average Age & 64.4 & 59.7 & 0.007 \\
\hline Male & 62 & 58 & 0.52 \\
\hline Female & 58 & 62 & 0.52 \\
\hline
\end{tabular}

There were five operations that were converted from the intended operation $(2.0 \%)$ and all were in the RR group. Three of the five operations converted to open were RR low anterior resections. The remaining two conversions were abdominoperineal resections (Figure 1). No conversions were necessary in the LR group.

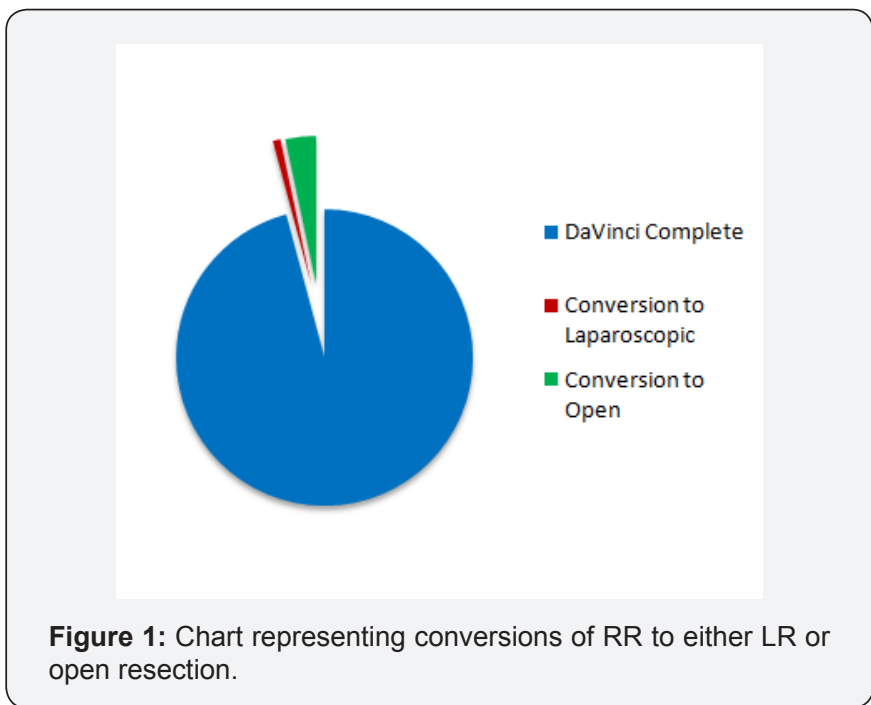
open resection.

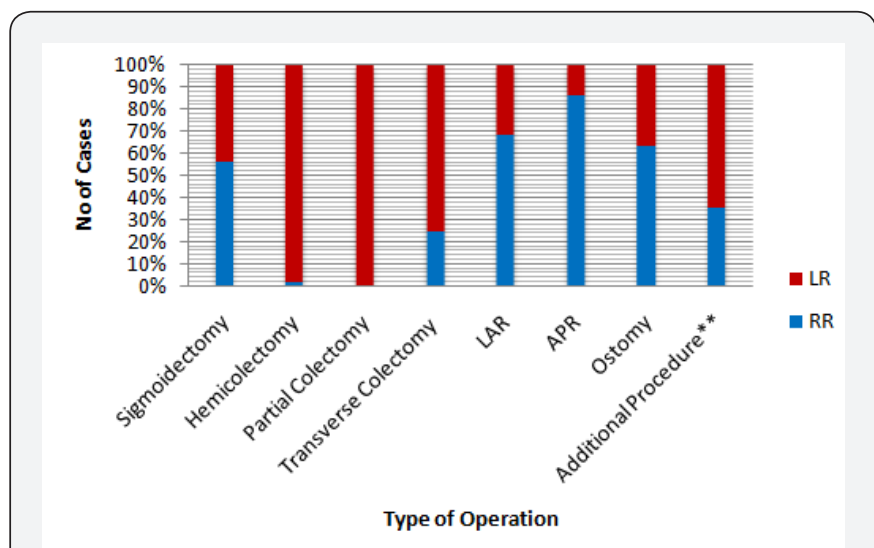

Figure 2: Comparing types of operations performed with LR vs.

More sigmoidectomies were performed with RR (RR 56.6 vs. LR $48.8 \mathrm{p}=0.19$ ). Hemicolectomies and partial (segmental) colectomies were performed more frequently in the LR group $(97.9,100.0,75.0, p=0.0001,0.08,0.32)$. LAR and APR were 
greater in the RR group $(68.5,86.7, p=0.0004,0.004)$. More ostomies were performed with $R R(63.6, p=0.37)$ but were not significantly different between the two groups. Additional procedures including appendectomy, cholecysctectomy, fistulectomy, abscess drainage, rectopexy, oophroectomy, and liver biopsy were also performed in addition to colon resection In certain cases with an appropriate indication (64.5 LR vs 35.5 $R R, p=0.11$ ) (Figure 2). Resections for malignancies were greater in the LR group ( $p=0.001)$ (Figures $3 \& 4)$. Of these operations, numbers of lymph nodes removed were similar between groups (LR 18.1 +/- 10.2 vs. RR 16.8 +/- 6.6, p=0.44) (Figure 5).

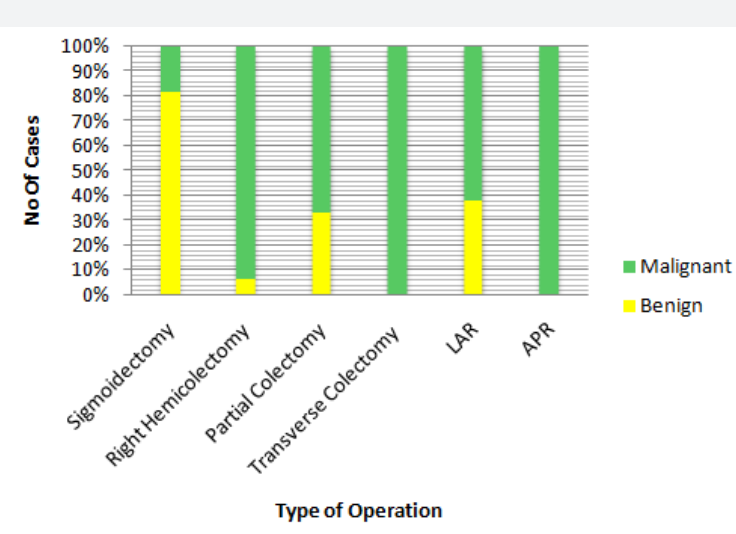

Figure 3: Comparing malignant vs benign pathology of various operations.

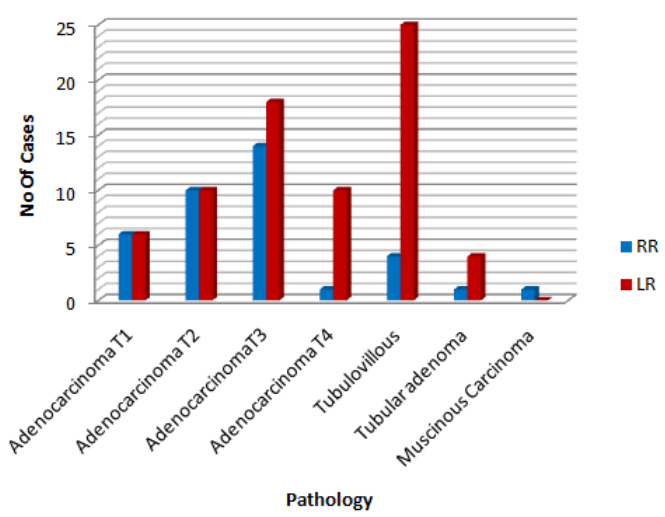

Figure 4: Comparing malignant pathology of LR vs RR.

The average operating room time for RR was longer compared to LR (151.6 min vs. $114 \mathrm{~min}, \mathrm{p}=0.0001$ ). The operating room time for RR of the first 30 cases averaged 189 min while the last 30 cases averaged $137 \mathrm{~min}(\mathrm{p}=0.0001)$ (Figure 6). The learning curve or RR appears to be around the first 60 cases (Figure 7). LR operating time was shorter, lasting $129 \mathrm{~min}$ for the first 30 cases and averaging $82 \mathrm{~min}$ for the last 30 cases.

There was no difference in the overall post-operative complication rate (LR $10.8 \%$ vs RR 6.7\%, p=0.36). Complications included post-operative bleed, pneumonia, anastomotic leak
(LR 1.7\% vs RR 1.6\%, p=0.53), wound infections, C. difficile infection; wound dehiscence and small bowel obstruction (Table 1, Figures 1-7).

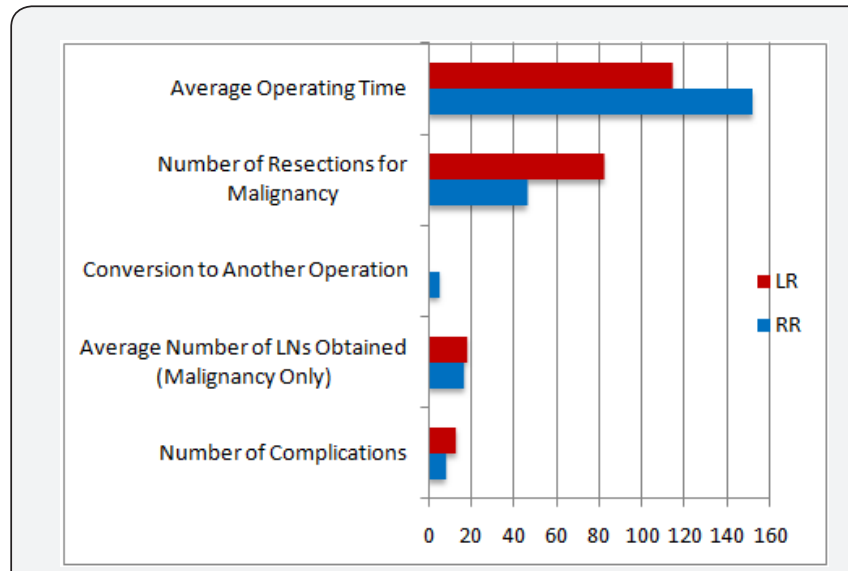

Figure 5: Comparing endpoints studied for LR vs RR.

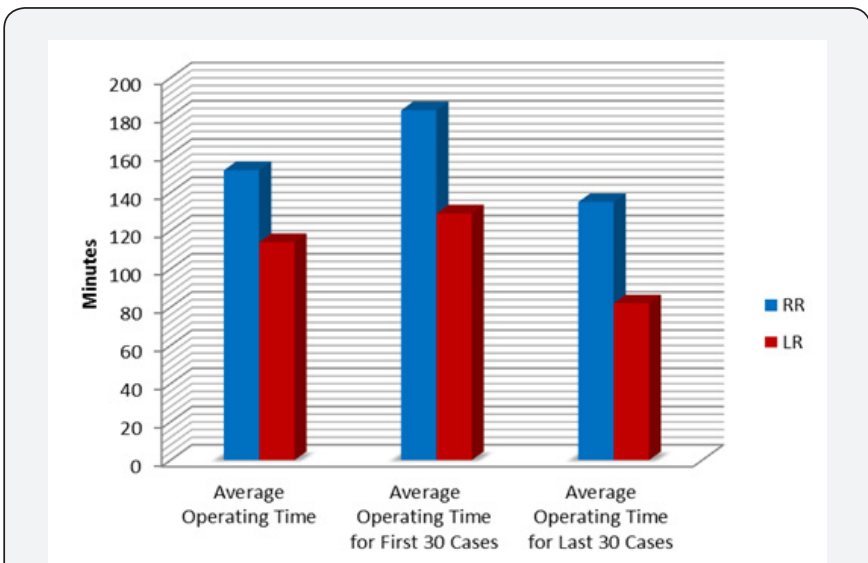

Figure 6: Comparing operative times for LR vs. RR.

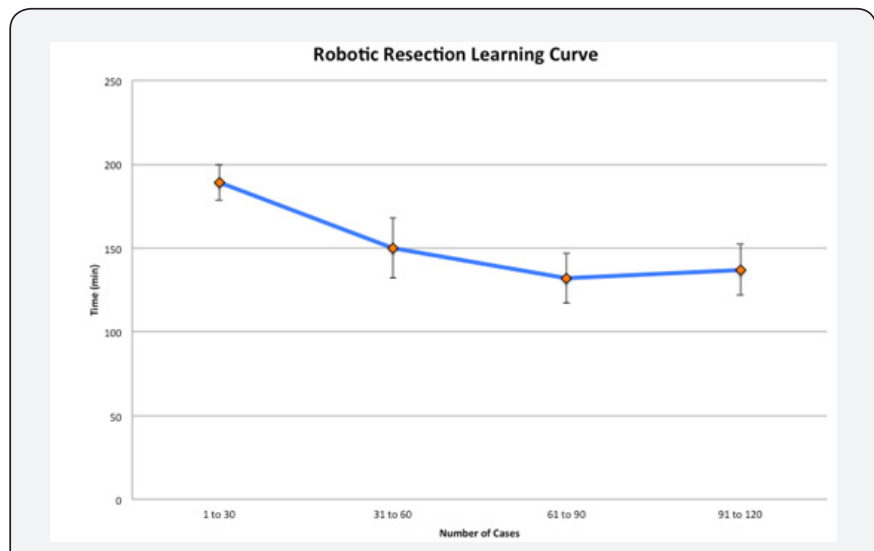

Figure 7: Graph to demonstrate learning curve for RR.

\section{Discussion}

Two types of colorectal resection (LR and RR) were compared for aforementioned variables. $2 \%$ conversion to another approach with robotic surgery (and even less than $2 \%$ 
conversion to open) shows that an adequately trained robotic surgeon can safely perform colorectal resections safely, as the rate of conversion to open in laparoscopic colorectal resections in a large, national retrospective review is $16.6 \%$.

LAR and APR were greater in the RR group (68.5, 86.7, $\mathrm{p}=0.0004,0.004)$, which is not surprising given the technical advantages of RR in the pelvis. Conversely, more ostomies were performed with $R R(63.6, p=0.37)$ but this is likely due to the fact that RR required low, tenuous anastomoses, potentially in a radiated field, which often require diversion.

Additional procedures including appendectomy, cholecysctectomy, fistulectomy, abscess drainage, rectopexy, oophroectomy, and liver biopsy were also performed in addition to colon resection mostly as planned operations. Preoperatively, decision for LR over RR for cases which involved additional procedures as described in Figure 2 was due to operating surgeon's experience with LR when resections required addressing other organ systems and/or involving other consulting surgeons (e.g Gynecology, Urology, etc).

Resections for malignancies were greater in the LR group $(\mathrm{p}=0.001)$ (Figures $3 \& 4$ ). Of these operations, numbers of lymph nodes harvested were similar between groups, showing that RR provides equivalency in oncologic colorectal resections. There was no difference in post-operative complication rate (LR $10.8 \%$ vs RR 6.7\%, p=0.36). Complications included postoperative bleeding, pneumonia, wound infections, C. difficile infection, wound dehiscence, need for unexpected transfusion, small bowel obstruction and most importantly anastomotic leaks (LR $1.7 \%$ vs RR 1.6\%, p=0.53). These graphs show that regardless of the surgical approach, the rates of complications are not statistically different.

Average operating room time for RR was longer compared to LR (151.6 min vs. $114 \mathrm{~min}, \mathrm{p}=0.0001$ ). This is an expected finding since LR has been the preferred method by the operating surgeon for over two decades. The operating room time for RR of the first 30 cases averaged 189 min while the last 30 cases averaged $137 \mathrm{~min}(\mathrm{p}=0.0001)$, which simply confirms that repetition is required for efficiency. The learning curve appears to be around the first 60 cases and this is thought to be due to the fact that not only the surgeon faces a steep technical learning curve but the operating room support staff also must adapt to the new technology, its intricate set-up, intra-operative troubleshooting and efficient turn over.

\section{Conclusion}

Our data confirms safety and efficacy of robotic colon resection for both benign and malignant disease when compared to laparoscopy. As it is widely practiced, laparoscopic rectal surgery is feasible, oncologically safe, and offers better shortterm outcomes than traditional open procedures in terms of pain control, recovery of bowel function, length of hospital stay, and time until return to working activity. However, laparoscopic techniques are not widely used in colorectal surgery due to prolonged and demanding learning curve that is available only in high-volume colorectal surgery centers experienced in minimally invasive colorectal surgery [5]. Barriers to laparoscopic techniques include unstable camera imaging, diminished dexterity, and a steep learning curve. For these reasons, widespread adoption of minimally invasive surgery techniques for colorectal disease is limited [6,7]. To overcome these technical drawbacks of laparoscopy, robotic surgical systems were introduced and reported in 2002 [8]. Robotic surgery is a new technology that enables the surgeon to perform minimally invasive operations with improved vision and control of the operating instruments. As such, robotic surgery may be a promising technology that can overcome some of the technical difficulties associated with standard laparoscopy [5].

Main advantages include stable camera platform, threedimensional imaging, excellent ergonomics, tremor elimination, ambidextrous capability, motion scaling and instruments with multiple degrees of freedom. With these advantages in mind, translation of the surgeon's hand movements into precise movements of the instruments inside the patient is achieved with exceptional control, the camera is held and entirely controlled by the operating surgeon, and a fourth robotic arm is available as a fixed retractor, minimizing the need for additional personnel in the operating room. All these aspects increase surgeon comfort and reduce fatigue [9]. Other advantages include smooth movements of the robotic arm, no tremors, motion scaling, a shorter learning curve [10], improved dexterity and ambidextrous capabilities [11-13] when compared to laparoscopy. Lastly, since major portion of the RR operations is performed while sitting down, the longevity of a robotic surgeon is likely to prove superior to open and laparoscopic techniques.

Therefore, the use of the robot is not only an acceptable alternative to laparoscopy but demonstrates superior technical advantages in certain colorectal operations and thus a multicenter, randomized, prospective analysis is encouraged to evaluate for potential survival advantage in malignant colorectal resections as well as a study to analyze the potential benefits of pelvic nerve preservation in benign and malignant colon and rectal resections.

\section{References}

1. Jacobs M, Verdeja JC, Goldstein HS (1991) Minimally invasive colon resection (laparoscopic colectomy). Surg Laparosc Endosc 1(3): 144150.

2. Kim JY, Kim NK, Lee KY, Hur H, Min BS, et al. (2012) A comparative study of voiding and sexual function after total mesorectal excision with autonomic nerve preservation for rectal cancer: laparoscopic versus robotic surgery. Ann Surg Oncol 19(8): 2485-2493.

3. Bianchi PP, Ceriani C, Locatelli A, Spinoglio G, Zampino MG, et al. (2010) Robotic versus laparoscopic total mesorectal excision for rectal cancer: a comparative analysis of oncological safety and short-term outcomes. Surg Endosc 24(11): 2888-2894. 
4. Rawlings AL, Woodland JH, Vegunta RK, Crawford DL (2007) Robotic versus laparoscopic colectomy. Surg Endosc 21(10): 1701-1708.

5. Bianchi PP, Luca F, Petz W, Valvo M, Cenciarelli S, et al. (2013) The role of the robotic technique in minimally invasive surgery in rectal cancer. Ecancermedicalscience 7: 357.

6. Liao G, Zhao Z, Lin S, Li R, Yuan Y, et al. (2014) Robotic-assisted versus laparoscopic colorectal surgery: a meta-analysis of four randomized controlled trials. World J Surg Oncol 12: 122.

7. Masoomi H, Moghadamyeghaneh Z, Mills S, Carmichael JC, Pigazzi A, et al. (2015) Risk factors for conversion of laparoscopic colorectal surgery to open surgery: does conversion worsen outcome? World J Surg 39(5):1240-1247.

8. Weber PA, Merola S, Wasielewski A, Ballantyne GH (2002) Teleroboticassisted laparoscopic right and sigmoid colectomies for benign disease. Dis Colon Rectum 45(12): 1689-1694.
9. Spinoglio G, Summa M, Priora F, Quarati R, Testa S (2009) Robotic laparoscopic surgery with the da Vinci® system: an early experience. Surg Technol Int 18: 70-74.

10. Jimenez-Rodriguez RM, Diaz-Pavon JM, de la Portilla DJF, PrendesSillero E, Dussort HC, et al. (2013) Learning curve for robotic-assisted laparoscopic rectal cancer surgery. Int J Colorectal Dis 28(6): 815-821.

11. D’Annibale A, Morpurgo E, Fiscon V, Trevisan P, Sovernigo G, et al. (2004) Robotic and laparoscopic surgery for treatment of colorectal diseases. Dis Colon Rectum 47(12): 2162-2168.

12. Park JS, Choi GS, Lim KH, Jang YS, Jun SH (2010) Robotic-assisted versus laparoscopic surgery for low rectal cancer: case-matched analysis of short-term outcomes. Ann Surg Oncol 17(12): 3195-3202.

13. Baik SH, Kwon HY, Kim JS, Hur H, Sohn SK, et al. (2009) Robotic versus laparoscopic low anterior resection of rectal cancer: short-term outcome of a prospective comparative study. Ann Surg Oncol 16(6): 1480-1487.

\section{Your next submission with Juniper Publishers will reach you the below assets}

- Quality Editorial service

- Swift Peer Review

- Reprints availability

- E-prints Service

- Manuscript Podcast for convenient understanding

- Global attainment for your research

- Manuscript accessibility in different formats

( Pdf, E-pub, Full Text, Audio)

- Unceasing customer service

Track the below URL for one-step submission https://juniperpublishers.com/online-submission.php 